\title{
Matrix variate Birnbaum-Saunders distribution under elliptical models
}

\author{
José A. Díaz-García * \\ Universidad Autónoma de Chihuahua \\ Facultad de Zootecnia y Ecología \\ Periférico Francisco R. Almada Km 1, Zootecnia \\ 33820 Chihuahua, Chihuahua, México \\ E-mail: jadiaz@uach.mx \\ Francisco J. Caro-Lopera \\ Departament of Basic Sciences \\ Universidad de Medellín \\ Medellín, Colombia \\ E-mail: fjcaro@udem.edu.co
}

\begin{abstract}
This paper derives the elliptical matrix variate version of the well known univariate Birnbaum and Saunders distribution. A generalisation based on a matrix transformation is proposed, instead of the independent element by element representation of the Gaussian univariate version of 1969. New results on Jacobians were needed to derived the matrix variate distribution. A number of special cases are studied and some basic properties are found. Finally, an example based on real data of two populations is provided. The maximum likelihood estimates are found for a number of matrix variate generalised Birnbaum-Saunders distributions based on Kotz models. A comparison with the Gaussian kernel is also given by using a modified BIC criterion.
\end{abstract}

\section{Introduction}

Some restricted situations in statistics accepts that the hypothesis for an experimental or observational data can be based on univariate tests. But the complex reality involves multivariate or matrix variate decision problems with several dependent variables that must be considered simultaneously.

This is the source of motivation to generalise the univariate probability distributions into the multivariate or matrix variate cases. However, the few known successful generalisations have required the creation of advanced mathematics, usually out of the scope of popular books and journals of high impact in decision sciences. Moreover, sometimes the leading techniques and the representations are not unique, then the associated theoretical relations

\footnotetext{
${ }^{*}$ Corresponding author

Key words. Matrix multivariate distributions, linear structures, random matrices, elliptical distributions, Birnbaum-Saunders distribution.

2000 Mathematical Subject Classification. 62E15; 60E05; 15B52
} 
enlarge the problem. For example, the extension of the univariate chi-squared into the so termed matrix variate Wishart distribution required the construction in the 50's of the theory of zonal polynomials of matrix arguments. Three different methods constructed the non singular central distribution: the singular value decomposition (SVD), the polar factorisation and the QR decomposition; see for example James (1954), Herz (1955) and Roy (1957), respectively. But their use in the computation of the joint latent roots distribution in the central case took more than 50 years after their apparition, and the relations among the densities are still unclear today. In fact, the theory for the extension to the non central Wishart was so advanced that the created invariant polynomials of several matrix arguments of Davis (1979) cannot be calculated even in this time of super computers.

Now, there are two ways to generalise a univariate random variable into a random vector or a random matrix:

i) Define the random vector or random matrix element by element.

ii) Propose a matrix transformation equivalent to the univariate function that defines the random variable $Y$.

For example, suppose a random variable $Y$ with a chi-square distribution of $n$ degrees of freedom, that is, $Y \sim \chi^{2}(n)$. Now, assume that the random vector $\mathbf{Z} \in \Re^{n}$ follows an $n$ dimensional normal distribution, with vector mean $\mathrm{E}(\mathbf{Z})=\mathbf{0}_{n} \in \Re^{n}$ and covariance matrix $\operatorname{Cov}(\mathbf{Z})=\mathbf{I}_{n}$; where $\mathbf{0}_{n}$ is a vector of zeros and $\mathbf{I}_{n}$ is the $n \times n$ identity matrix. In notation, $\mathbf{Z} \sim \mathcal{N}_{n}\left(\mathbf{0}_{n}, \mathbf{I}_{n}\right)$. Then, we know that $Y \stackrel{d}{=}\|\mathbf{Z}\|^{2}$; where $\stackrel{d}{=}$ holds for equally distributed and $\|\mathbf{Z}\|$ denotes the Euclidiana norm of the vector $\mathbf{Z}$. So, we ask for the multivariate version of the random variable $Y$.

Applying the first method (element-to-element) we can proceed as follows: let $\mathbf{Z} \sim$ $\mathcal{N}_{n}\left(\mathbf{0}_{n}, \mathbf{I}_{n}\right)$, such that $n=n_{1}+n_{2}$ and $\mathbf{Z}^{\prime}=\left(\mathbf{Z}_{1}^{\prime}, \mathbf{Z}_{2}^{\prime}\right), \mathbf{Z}_{1} \in \Re^{n_{1}}$ and $\mathbf{Z}_{2} \in \Re^{n_{2}}$. Then, define the random variables $Y_{i}=\left\|\mathbf{Z}_{i}\right\|^{2}, i=1,2$ and the vector random $\mathbf{Y}^{\prime}=\left(Y_{1}, Y_{2}\right)^{\prime}$. Thus $\mathbf{Y}$ is said to have a bidimensional $\chi^{2}$ distribution, such that, $Y_{i} \sim \chi^{2}\left(n_{i}\right), i=1,2$; see Libby and Novick (1982). Using the same technique we can get the multivariate version of the random variable $Y$. Sometimes the matrix case can be obtained directly from multivariate (vector) version: let $\mathbf{Y} \in \Re^{n \times m}$ and define $\mathbf{v}=\operatorname{vec}(\mathbf{Y})$, where $\operatorname{vec}(\mathbf{Y})$ denotes de vectorisation of the matrix $\mathbf{Y}$, then the distribution of $\mathbf{Y}$ is obtained from the distribution of the random vector $\mathbf{v}$.

Alternatively, the matrix variate extension of the $\chi^{2}$-distribution became more popular that the addressed multivariate case. Assume $n$ independent $\mathbf{Z}_{i} \sim \mathcal{N}_{m}(\mathbf{0}, \boldsymbol{\Sigma})$, with $\operatorname{Cov}\left(\mathbf{Z}_{i}\right)=\boldsymbol{\Sigma}$ and $i=1, \ldots, n$. Define the random matrix

$$
\mathbf{V}=\sum_{i=1}^{n} \mathbf{Z}_{i} \mathbf{Z}_{i}^{\prime}
$$

If $n \geq m$, then $\mathbf{V}$ is positive definite $(\mathbf{V}>\mathbf{0})$ and $\mathbf{V}$ is said to have a Wishart distribution. Otherwise, if $n<m$, then $\mathbf{V}$ is positive semidefinite, $(\mathbf{V} \geq \mathbf{0})$ and $\mathbf{V}$ is said to have a pseudoWishart distribution. These facts are denoted as $\mathbf{V} \sim \mathcal{W}_{m}(n, \boldsymbol{\Sigma})$ and $\mathbf{V} \sim \mathcal{P} \mathcal{W}_{m}(n, \boldsymbol{\Sigma})$, respectively, see Srivastava and Khatri (1979) and Muirhead (2005), among many others. Note that, if $m=1, \boldsymbol{\Sigma}$ is an scalar, say $\sigma^{2}$, then $n \mathbf{V} / \sigma^{2} \equiv n Y / \sigma^{2} \sim \chi^{2}(n)$. However, note that it is impossible to obtain a vector version of the distribution of $\mathbf{V}$ from the Wishart distribution. In addition, not all elements $v_{i j}$ (in $\mathbf{V}$ ) follow a $\chi^{2}$-distribution. Note that, if the univariate random variable is a function of square or square root operators, the corresponding matrix variate version via a matrix transformation, must be a random square matrix; moreover, in general, it must be a random symmetric matrix, see Cadet (1996), Olkin and Rubin (1964), Muirhead (2005), and references therein. Then, the matrix 
version includes the univariate case, but the vector version cannot be derived, moreover, the elements of the matrix does not follow the original univariate distribution.

However, a matrix variate version via element-to-element has not order constraint. The vectorial and the univariate cases can be derived directly from the matrix case, and all the elements of the matrix have as marginal distribution, the original univariate distribution, see Chen and Novick (1984) and Libby and Novick (1982).

Extreme unusual cases allows equivalence among the vector version and the elementto-element representation and the matrix transformation. This occurs in the multivariate t-distribution; which is a consequence of a property for the t-distribution family, see Kotz and Nadarajah (2004, p. 2, 4). A random $p$-dimensional vector with distribution $t$ can be defined in two ways:

$$
\mathbf{t}=\left\{\begin{array}{c}
S^{-1} \mathbf{Y}+\boldsymbol{\mu}=\left(\begin{array}{c}
y_{1} / S^{-1}+\mu_{1} \\
y_{2} / S^{-1}+\mu_{2} \\
\vdots \\
y_{p} / S^{-1}+\mu_{p}
\end{array}\right), \\
\text { with } \frac{\nu S^{2}}{\sigma^{2}} \sim \chi^{2}(\nu) \text { and } \mathbf{Y} \sim \mathcal{N}_{p}(\mathbf{0}, \boldsymbol{\Sigma}) ; \\
\mathbf{W}^{-1 / 2} \mathbf{Y}+\boldsymbol{\mu}, \quad \text { with } \quad \mathbf{W} \sim \mathcal{W}_{p}(\nu+p-1, \boldsymbol{\Sigma}) \text { and } \mathbf{Y} \sim \mathcal{N}_{p}\left(\mathbf{0}, \nu \mathbf{I}_{p}\right) .
\end{array}\right.
$$

with $\left(\mathbf{W}^{1 / 2}\right)^{2}=\mathbf{W}$ and $\boldsymbol{\mu}: p \times 1$ a constant vector.

Nevertheless, this unusual property is not fulfilled in the matrix case. Consider the sample $\mathbf{t}_{1}, \ldots, \mathbf{t}_{n}$ of a multivariate population with $\mathbf{t}$ distribution, and consider the matrix $\mathbf{T}=\left(\mathbf{t}_{1} \cdots \mathbf{t}_{n}\right): p \times n$, then

$$
\mathbf{T}=\left\{\begin{array}{c}
\left(\begin{array}{c}
S^{-1} \mathbf{Y}_{1}^{T}+\boldsymbol{\mu}_{1}^{T} \\
\vdots \\
S^{-1} \mathbf{Y}_{n}^{T}+\boldsymbol{\mu}_{n}^{T}
\end{array}\right)^{T}=S^{-1} \mathbb{Y}+\mathbf{M}, \\
\text { with } \quad \frac{\nu S^{2}}{\sigma^{2}} \sim \chi^{2}(\nu) \text { and } \mathbb{Y} \sim \mathcal{N}_{p \times n}\left(\mathbf{0}, \boldsymbol{\Sigma} \otimes \mathbf{I}_{n}\right) \\
\text { or } \left.\begin{array}{c}
\mathbf{Y}_{1}^{T} \mathbf{W}^{-1 / 2}+\boldsymbol{\mu}_{1}^{T} \\
\vdots \\
\mathbf{Y}_{n}^{T} \mathbf{W}^{-1 / 2}+\boldsymbol{\mu}_{n}^{T} \\
\text { with }
\end{array}\right)^{T}=\mathbf{W}^{-1 / 2} \mathbb{Y}+\mathbf{M}, \\
\end{array}\right.
$$

where $\mathbf{M}=\left(\boldsymbol{\mu}_{1} \cdots \boldsymbol{\mu}_{n}\right): p \times n$, and $\mathbb{Y}=\left(\mathbf{Y}_{1} \cdots \mathbf{Y}_{n}\right)$. But the random matrix $\mathbf{T}$ does not have the same distribution under the above two representations, even when their rows have the same distribution. In the first representation, $\mathbf{T}$ has a matrix multivariate $t$-distribution and under the second one it has a matricvariate T-distribution, see Kotz and Nadarajah (2004, p. 2, 4). Also, note that the matricvariate $T$-distribution cannot be obtained from the matrix-variate $t$-distribution, and vice versa.

Now we focus on the distribution of this work. An important lifetime model was introduced by Birnbaum and Saunders (1969) in the context of a problem of material fatigue. The so termed Birnbaum-Saunders distribution is a lifetime model for fatigue failure caused under cyclic loading and assumed that the failure is due to the development and growth of a dominant crack. A more general derivation was provided by Desmond (1985) based on a biological model.

The original univariate random variable was supported by a normal distribution, then 
the so termed Gaussian Birnbaum-Saunders random variable $T$ is the distribution of

$$
T=\beta\left(\frac{\alpha}{2} Z+\sqrt{\left(\frac{\alpha}{2} Z\right)^{2}+1}\right)^{2},
$$

where $Z \sim \mathcal{N}(0,1)$. We shall denotes this fact as $T \sim \mathcal{B S}(\alpha, \beta)$, where $\alpha>0$ is the shape parameter, and $\beta>0$ is both scale parameter an the median value of the distribution. Then, the inverse relation establishes that if $T \sim \mathcal{B S}(\alpha, \beta)$, then

$$
Z=\frac{1}{\alpha}\left(\sqrt{\frac{T}{\beta}}-\sqrt{\frac{\beta}{T}}\right) \sim \mathcal{N}(0,1)
$$

Díaz-García and Leiva-Sánchez (2005, 2006) propose a generalisation of the Birnbaum-Saunders distribution, replacing the Gaussian hypothesis in (2) by a symmetric distribution, i.e. they assume that $Z \sim \mathcal{E}(0,1, h)$. We recall that the density function of $Z \sim \mathcal{E}(0,1, h)$ is defined as $f_{Z}(z)=h\left(z^{2}\right)$, for $z \in \Re$. Therefore, (1) defines the so termed generalised BirnbaumSaunders distribution, which shall be denoted by $T \sim \mathcal{G B S}(\alpha, \beta ; h)$. Note the long delay to appear the elliptical univariate version. In fact, the element-to-element elliptical matrix variate version of Birnbaum and Saunders (1969) was published in Caro-Lopera et al. (2012), it demanded the develop of some theory to connect the Hadamard product and the usual matrix product. In the same direction, Caro-Lopera and Díaz-García (2016) studied the so termed diagonalisation matrix and applied it in another matrix representation of the element-to-element matrix variate generalised Birnbaum-Saunders distribution. Moreover, Sánchez et al. (2015) performed estimation for the matrix parameters of that type matrix case. But a matrix transformation has been so elusive in literature and no clue to derive such transformation can be inferred or proposed from the existing extensions of another families matrix variate distributions. The importance of the Birnbaum-Saunders distribution is indisputable, recently Balakrishnan and Kundu (2018) make a detailed compilation of this distribution. That review of 108 pages and 281 references, describes widely and profusely the univariate and multivariate cases in a long history since the 60 's, however the very short history of the element-by-element version of the matrix variate case was covered in only 1 of such references. Two new references about the element-by-element version, can be seen in Caro-Lopera et al. (2012) and Sánchez et al. (2015).

Finally, we addressed that the differences between the two GBS versions (the proposed matrix transformation version and the published element-by-element version) can be highlighted in two important issues: First, both matrix versions have only one aspect in common, they include the univariate generalised Birnbaum-Saunders distribution as a particular case. However, for higher dimensions the new version provides a natural way of introducing matrix distributions from the univariate case. The element-by-element representation was the first attempt to attack the problem, but a version based on a matrix transformation was elusive for more than 50 years. The key point for the solution of the problem can be simplified in the next table. Finally, the proposed matrix version allows the use of the classical matrix variate distribution theory, matrix transformations and general inference, because it is set in terms on matrices, instead of the elements of the matrix.

\begin{tabular}{c|c}
\hline \hline BS published & BS proposed \\
\hline \hline element-by-element & matrix transformation \\
\hline rectangular matrix & square matrix \\
\hline- & positive definite matrix \\
\hline
\end{tabular}

This paper compute some new Jacobians in order to derive the matrix variate BirnbaumSaunders distribution under elliptical models. Some basic properties are studied and the 
expected corollaries are derived. For a real database, the article concludes obtaining the maximum likelihood estimators of the parameters of a matrix variate generalised BirnbaumSaunder distribution which is based on the matrix variate Kotz distribution.

Then, the paper is organised as follows: in Section 2 some preliminary results and new Jacobians are provided. Section 3 derives the main result of the paper. Some basic properties are studied and the expected corollaries are derived. Finally, Section 4 studies the parameter estimation and a comparison of some Birnbaum-Saunders distributions based on a Kotz type elliptical model, which includes the Gaussian case.

\section{Preliminary results}

Some properties and definitions in matrix variate elliptical theory are summarised below. A detailed study of this family of distributions is presented in Fang and Zhang (1990) and Gupta et al. (2013), among many others authors. This section also presents the published element-to-element representations of the Birnbaum-Saunders distribution and new Jacobians are computed. First, some results and notations about the required matrix algebra are considered, see Rao (2005) and Muirhead (2005).

\subsection{Notation}

For our purposes: if $\mathbf{A} \in \Re^{n \times m}$ denotes a matrix, this is, $\mathbf{A}$ have $n$ rows and $m$ columns, then $\mathbf{A}^{\prime} \in \Re^{m \times n}$ denotes its transpose matrix, and if $\mathbf{A} \in \Re^{n \times n}$ has an inverse, it shall be denoted by $\mathbf{A}^{-1} \in \Re^{n \times n}$. An identity matrix shall be denoted by $\mathbf{I} \in \Re^{n \times n}$, to specified the size of the identity, we shall use $\mathbf{I}_{n}$. A null matrix shall be denoted as $\mathbf{0} \equiv \mathbf{0}_{n \times m} \in \Re^{n \times m}$. For all matrix $\mathbf{A} \in \Re^{n \times m}$ exist $\mathbf{A}^{+} \in \Re^{m \times n}$ which is termed Moore-Penrose inverse. The eigenvalues of $\mathbf{A} \in \Re^{n \times n}$ are the roots of the equation $\left|\mathbf{A}-\lambda \mathbf{I}_{n}\right|=0 . \mathbf{A} \in \Re^{n \times n}$ is a symmetric matrix if $\mathbf{A}=\mathbf{A}^{\prime}$ and if all their eigenvalues are positive then $\mathbf{A}$ is positive definite matrix, which shall be denoted as $\mathbf{A}>\mathbf{0}$. The $i-t h$ eigenvalue of $\mathbf{A}$ shall be denoted as $\operatorname{ch}_{i}(\mathbf{A})$. Given a definite positive matrix $\mathbf{A} \in \Re^{m \times m}$, there exist a positive definite matrix $\mathbf{A}^{1 / 2} \in \Re^{m \times m}$ such that $\mathbf{A}=\left(\mathbf{A}^{1 / 2}\right)^{2}$, which is termed positive definite root matrix. The set of matrices $\mathbf{H}_{1} \in \Re^{n \times m}$ such that $\mathbf{H}_{1}^{\prime} \mathbf{H}_{1}=\mathbf{I}_{m}$ is a manifold denoted $\mathcal{V}_{m, n}$, termed Stiefel manifold. In particular, $\mathcal{V}_{m, m}$ is the group of orthogonal matrices $\mathcal{O}(m)$. If $\mathbf{A} \in \Re^{n \times m}$ is writing in terms of its $m$ columns, $\mathbf{A}=\left(\mathbf{A}_{1}, \mathbf{A}_{2}, \ldots, \mathbf{A}_{m}\right), \mathbf{A}_{j} \in \Re^{n}, j=1,2 \ldots, m, \operatorname{vec}(\mathbf{A}) \in \Re^{n m}$ denotes the vectorisation of $\mathbf{A}$, moreover, $\operatorname{vec}^{\prime}(\mathbf{A})=(\operatorname{vec}(\mathbf{A}))^{\prime}=\left(\mathbf{A}_{1}^{\prime}, \mathbf{A}_{2}^{\prime}, \ldots, \mathbf{A}_{m}^{\prime}\right)$. Let $\mathbf{A} \in \Re^{r \times s}$ and $\mathbf{B} \in \Re^{n \times m}$, then $\mathbf{A} \otimes \mathbf{B} \in \Re^{s n \times r m}$ denotes its Kronecker product. For $\mathbf{A}, \mathbf{B}$, and $\mathbf{C}$, matrices of suitable matrices orders, we have

$$
\operatorname{vec}(\mathbf{A B C})=\left(\mathbf{C}^{\prime} \otimes \mathbf{A}\right) \operatorname{vec} \mathbf{B} .
$$

The commutative matrix $\mathbf{K}_{n m} \in \Re^{n m \times n m}$ is the matrix with the property that $\mathbf{K}_{n m}$ vec $\mathbf{A}=$ vec $\mathbf{A}^{\prime}$, for every matrix $\mathbf{A} \in \Re^{n \times m}$. In addition for $\mathbf{A} \in \Re^{m \times m}$, and $\mathbf{B} \in \Re^{p \times q}$,

$$
\mathbf{K}_{p m}(\mathbf{A} \otimes \mathbf{B})=(\mathbf{B} \otimes \mathbf{A}) \mathbf{K}_{q n} .
$$

\subsection{Matrix variate distribution.}

Definition 2.1. Is said that $\mathbf{Y} \in \Re^{n \times m}$ has a matrix variate elliptically contoured distribution if its density with respect to the Lebesgue measure is given by:

$$
d F_{\mathbf{Y}}(\mathbf{Y})=\frac{1}{|\boldsymbol{\Sigma}|^{n / 2}|\boldsymbol{\Theta}|^{m / 2}} h\left\{\operatorname{tr}\left[(\mathbf{Y}-\boldsymbol{\mu})^{\prime} \boldsymbol{\Theta}^{-1}(\mathbf{Y}-\boldsymbol{\mu}) \boldsymbol{\Sigma}^{-1}\right]\right\}(d \mathbf{Y}),
$$


where $\boldsymbol{\mu} \in \Re^{n \times m}, \boldsymbol{\Sigma} \in \Re^{m \times m}, \boldsymbol{\Theta} \in \Re^{n \times n}, \boldsymbol{\Sigma}>\mathbf{0}$ and $\boldsymbol{\Theta}>\mathbf{0}$ and $(d \mathbf{Y})$ is the Lebesgue measure. The function $h: \Re \rightarrow[0, \infty)$ is termed the generator function and satisfies $\int_{0}^{\infty} u^{m n-1} h\left(u^{2}\right) d u<\infty$. Such a distribution is denoted by $\mathbf{Y} \sim \mathcal{E}_{n \times m}(\boldsymbol{\mu}, \boldsymbol{\Theta} \otimes \boldsymbol{\Sigma}, h)$, see Gupta et al. (2013).

When $\boldsymbol{\mu}=\mathbf{0}_{n \times m}, \boldsymbol{\Sigma}=\mathbf{I}_{m}$ and $\boldsymbol{\Theta}=\mathbf{I}_{n}$, such distribution is termed matrix variate symmetric distribution and shall be denoted as $\mathbf{Y} \sim \mathcal{E}_{n \times m}\left(\mathbf{0}, \mathbf{I}_{n m}, h\right)$.

Observe that this class of matrix variate distributions includes normal, contaminated normal, Pearson type II and VI, Kotz, logistic, power exponential, and so on; these distributions have tails that are weighted more or less, and/or they have greater or smaller degree of kurtosis than the normal distribution.

From Díaz-García and Leiva-Sánchez (2005, 2006) if $T \sim \mathcal{G B S}(\alpha, \beta, h)$, then

$$
d F_{T}(t)=\frac{t^{-3 / 2}(t+\beta)}{2 \alpha \sqrt{\beta}} h\left[\frac{1}{\alpha^{2}}\left(\frac{t}{\beta}+\frac{\beta}{t}-2\right)\right] d t, \quad t>0 .
$$

Alternatively, let $V=\sqrt{T}$, with $d t=2 v d v$, then under a symmetric distribution, (2) can be rewrite as

$$
Z=\frac{1}{\alpha}\left(\frac{V}{\sqrt{\beta}}-\frac{\sqrt{\beta}}{V}\right)
$$

and its density is given by

$$
d F_{V}(v)=\frac{\left(1+\beta v^{-2}\right)}{\alpha \sqrt{\beta}} h\left[\frac{1}{\alpha^{2}}\left(\frac{v^{2}}{\beta}+\frac{\beta}{v^{2}}-2\right)\right] d v, \quad v>0,
$$

which shall be termed square root generalised Birnbaum-Saunders distribution.

Among other authors, Díaz-García and Domínguez Molina (2006) proposed a multivariate version (vector version) defined element-to-element of the density function (5), this is, they assumed that $\mathbf{z} \sim \mathcal{E}_{n}\left(\mathbf{0}_{n}, \mathbf{I}_{n} ; h\right)$ and define the change of variable

$$
t_{i}=\beta_{i}\left(\frac{1}{2} \alpha_{i} z_{i}+\sqrt{\left(\frac{1}{2} \alpha_{i} z_{i}\right)^{2}+1}\right)^{2}, \quad \alpha_{i}>0, \quad \beta_{i}>0, \quad i=1, \ldots, n
$$

Then, the density $d F_{\mathbf{t}}\left(t_{1}, \ldots, t_{n}\right)$ of $\mathbf{t}=\left(t_{1}, \ldots, t_{n}\right)^{\prime} \in \Re_{+}^{n}$, termed multivariate generalised Birnbaum-Saunders distribution, is given by

$$
=\frac{1}{2^{n}}\left(\prod_{i=1}^{n} \frac{t_{i}^{-3 / 2}\left(t_{i}+\beta_{i}\right)}{\alpha_{i} \sqrt{\beta_{i}}}\right) h\left[\sum_{i=1}^{n} \frac{1}{\alpha_{i}^{2}}\left(\frac{t_{i}}{\beta_{i}}+\frac{\beta_{i}}{t_{i}}-2\right)\right]\left(\bigwedge_{i=1}^{n} d t_{i}\right),
$$

where $\bigwedge$ denotes the exterior product, see Muirhead (2005, Section 2.1.1, p. 50). This fact is denoted as $\mathbf{t} \sim \mathcal{G B S}_{n}(\boldsymbol{\alpha}, \boldsymbol{\beta} ; h)$, with $\boldsymbol{\alpha}=\left(\alpha_{1}, \ldots, \alpha_{n}\right)^{\prime}$ and $\boldsymbol{\beta}=\left(\beta_{1}, \ldots, \beta_{n}\right)^{\prime}$. This distribution was studied in detail by Díaz-García and Domínguez Molina (2007) when $\beta_{1}=$ $\cdots=\beta_{n}=\beta$ and $\alpha_{1}=\cdots=\alpha_{n}=\alpha$.

As we mentioned above, the matrix variate generalised Birbaum-Saunders distribution can be obtained from the multivariate case by defining the vector $\mathbf{r}=\operatorname{vec} \mathbf{T}$, where $\mathbf{T} \in$ $\Re^{n \times m}$ and

$$
t_{i j}=\beta_{i j}\left(\frac{1}{2} \alpha_{i j} z_{i j}+\sqrt{\left(\frac{1}{2} \alpha_{i j} z_{i j}\right)^{2}+1}\right)^{2}, \quad \alpha_{i j}>0, \quad \beta_{i j}>0
$$


with $i=1, \ldots, n ; j=1, \ldots, m$. Then, assuming that $\mathbf{Z} \sim \mathcal{E}_{n \times m}\left(\mathbf{0}, \mathbf{I}_{n m}, h\right)$ the density $D F_{\mathbf{T}}\left(\left(t_{i j}\right)_{j=1, \ldots, m}^{i=1, \ldots, n}\right), t_{i j}>0$ is given by

$$
=\frac{1}{2^{n m}}\left(\prod_{i=1}^{n} \prod_{j=1}^{m} \frac{t_{i j}^{-3 / 2}\left(t_{i j}+\beta_{i j}\right)}{\alpha_{i j} \sqrt{\beta_{i j}}}\right) h\left[\sum_{i=1}^{n} \sum_{j=1}^{m} \frac{1}{\alpha_{i j}^{2}}\left(\frac{t_{i j}}{\beta_{i j}}+\frac{\beta_{i j}}{t_{i j}}-2\right)\right]\left(\bigwedge_{i=1}^{n} \bigwedge_{j=1}^{m} d t_{i j}\right),
$$

which is denoted as $\mathbf{T} \sim \mathcal{G B S}_{n \times m}(\mathbf{A}, \mathbf{B} ; h)$, with $\mathbf{A}=\left(\alpha_{i j}\right)$, and $\mathbf{B}=\left(\beta_{i j}\right), i=1, \ldots, n$; $j=1, \ldots, m$.

This distribution was found and studied by Caro-Lopera et al. (2012). Their main goal was to construct a matrix representation of the matrix variate generalised BirnbaumSaunders distribution. Using the diagonalisation operator, the Hadamard product and partition theory, they propose two matrix representations of the density function (9). In terms of the diagonalisation matrix, an alternative matrix representation of the matrix variate

generalised Birnbaum-Saunders distribution was proposed by Caro-Lopera and Díaz-García (2016).

\section{$2.3 \quad$ Jacobians}

Theorem 2.1. Consider the follow matrix transformation

$$
\mathbf{Z}=\left(\mathbf{V} \boldsymbol{\Delta}^{-1}-\mathbf{V}^{\prime+} \boldsymbol{\Delta}\right) \mathbf{\Xi}^{-1}
$$

where $\mathbf{Z}$ and $\mathbf{V} \in \Re^{n \times m}$ with element functionally independent and both of rank $m \leq n, \boldsymbol{\Delta}$ and $\boldsymbol{\Xi} \in \Re^{m \times m}$, with $\boldsymbol{\Delta}>\mathbf{0}$ and $\boldsymbol{\Xi}>\mathbf{0}$. Then

$$
\begin{aligned}
(d \mathbf{Z})= & |\boldsymbol{\Xi}|^{-n} \mid \boldsymbol{\Delta}^{-1} \otimes \mathbf{I}_{n}+\left(\boldsymbol{\Delta} \otimes \mathbf{I}_{n}\right)\left[\mathbf{K}_{m n}\left(\mathbf{V}^{\prime} \otimes \mathbf{V}^{+}\right)\right. \\
& \left.-\left(\mathbf{V}^{\prime} \mathbf{V}\right)^{-1} \otimes\left(\mathbf{I}_{n}-\mathbf{V} \mathbf{V}^{+}\right)\right] \mid(d \mathbf{V}) .
\end{aligned}
$$

Proof. Let

$$
\mathbf{Z}=\left(\mathbf{V} \boldsymbol{\Delta}^{-1}-\mathbf{V}^{\prime+} \boldsymbol{\Delta}\right) \mathbf{\Xi}^{-1}
$$

To determine the Jacobian under the change of variable (10), we shall proceed using the theory developed by Magnus (1988) and Magnus and Neudecker (2007). For $\mathbf{X} \in \Re^{n \times m}$ by Magnus and Neudecker (2007, Theorem 5, p. 174) it is known that

$$
d \mathbf{X}^{+}=-\mathbf{X}^{+} d \mathbf{X} \mathbf{X}^{+}+\mathbf{X}^{+} \mathbf{X}^{+^{\prime}} d \mathbf{X}^{\prime}\left(\mathbf{I}_{n}-\mathbf{X} \mathbf{X}^{+}\right)+\left(\mathbf{I}_{m}-\mathbf{X}^{+} \mathbf{X}\right) d \mathbf{X}^{\prime} \mathbf{X}^{+^{\prime}} \mathbf{X}^{+},
$$

also recalling that $d \mathbf{A X B}=\mathbf{A} d \mathbf{X B}$; and observing that in our case $\mathbf{V}^{+} \mathbf{V}=\mathbf{I}_{m},[d \mathbf{V}]^{\prime}=$ $d \mathbf{V}^{\prime},\left(\mathbf{I}_{n}-\mathbf{V} \mathbf{V}^{+}\right)=\left(\mathbf{I}_{n}-\mathbf{V} \mathbf{V}^{+}\right)^{\prime},\left(\mathbf{V}^{+}\right)^{\prime}=\mathbf{V}^{+^{\prime}}=\mathbf{V}^{\prime}+$ and $\left(\mathbf{V}^{\prime} \mathbf{V}\right)^{+}=\left(\mathbf{V}^{\prime} \mathbf{V}\right)^{-1}$. Hence taking differentials in (10) we have

$$
\begin{aligned}
d \mathbf{Z} & =\left(d \mathbf{V} \boldsymbol{\Delta}^{-1}-d \mathbf{V}^{+^{\prime}} \boldsymbol{\Delta}\right) \boldsymbol{\Xi}^{-1} \\
& =d \mathbf{V} \boldsymbol{\Delta}^{-1} \boldsymbol{\Xi}^{-1}-\left[-\mathbf{V}^{+} d \mathbf{V} \mathbf{V}^{+}+\left(\mathbf{V}^{\prime} \mathbf{V}\right)^{-1} d \mathbf{V}^{\prime}\left(\mathbf{I}_{n}-\mathbf{V} \mathbf{V}^{+}\right)\right]^{\prime} \boldsymbol{\Delta} \boldsymbol{\Xi}^{-1} \\
& =d \mathbf{V} \boldsymbol{\Delta}^{-1} \boldsymbol{\Xi}^{-1}+\mathbf{V}^{{ }^{\prime}} d \mathbf{V}^{\prime} \mathbf{V}^{+} \boldsymbol{\Delta}^{-1}-\left(\mathbf{I}_{n}-\mathbf{V} \mathbf{V}^{+}\right) d \mathbf{V}\left(\mathbf{V}^{\prime} \mathbf{V}\right)^{-1} \boldsymbol{\Delta} \boldsymbol{\Xi}^{-1}
\end{aligned}
$$

By vectorisation, we get

$$
\begin{aligned}
d \operatorname{vec} \mathbf{Z}= & \left(\left(\boldsymbol{\Delta}^{-1} \boldsymbol{\Xi}^{-1}\right)^{\prime} \otimes \mathbf{I}_{n}\right) d \operatorname{vec} \mathbf{V}+\left[\left(\mathbf{V}^{\prime}+\boldsymbol{\Delta} \boldsymbol{\Xi}^{-1}\right)^{\prime} \otimes \mathbf{V}^{\prime}+\right] d \operatorname{vec} \mathbf{V}^{\prime} \\
& -\left[\left(\left(\mathbf{V}^{\prime} \mathbf{V}\right)^{-1} \boldsymbol{\Delta} \boldsymbol{\Xi}^{-1}\right)^{\prime} \otimes\left(\mathbf{I}_{n}-\mathbf{V} \mathbf{V}^{+}\right)\right] d \operatorname{vec} \mathbf{V}
\end{aligned}
$$


Therefore, given that $\boldsymbol{\Xi}$ and $\boldsymbol{\Delta}$ are symmetric matrices, we obtain

$$
\begin{aligned}
d \operatorname{vec} \mathbf{Z}= & \left(\boldsymbol{\Xi}^{-1} \boldsymbol{\Delta}^{-1} \otimes \mathbf{I}_{n}+\left(\boldsymbol{\Xi}^{-1} \boldsymbol{\Delta} \mathbf{V}^{+} \otimes \mathbf{V}^{\prime}+\right) \mathbf{K}_{n m}\right. \\
& \left.-\boldsymbol{\Xi}^{-1} \boldsymbol{\Delta}\left(\mathbf{V}^{\prime} \mathbf{V}\right)^{-1} \otimes\left(\mathbf{I}_{n}-\mathbf{V} \mathbf{V}^{+}\right)\right) d \operatorname{vec} \mathbf{V}
\end{aligned}
$$

In addition, noting that $(\mathbf{A B} \otimes \mathbf{C D})=(\mathbf{A} \otimes \mathbf{C})(\mathbf{B} \otimes \mathbf{D})$ we get

$$
\begin{aligned}
d \operatorname{vec} \mathbf{Z}= & \left(\boldsymbol{\Xi}^{-1} \otimes \mathbf{I}_{n}\right)\left\{\boldsymbol{\Delta}^{-1} \otimes \mathbf{I}_{n}+\left(\boldsymbol{\Delta} \otimes \mathbf{I}_{n}\right)\left[\mathbf{K}_{m n}\left(\mathbf{V}^{+^{\prime}} \otimes \mathbf{V}^{+}\right)\right.\right. \\
& \left.\left.-\left(\mathbf{V}^{\prime} \mathbf{V}\right)^{-1} \otimes\left(\mathbf{I}_{n}-\mathbf{V} \mathbf{V}^{+}\right)\right]\right\} d \operatorname{vec} \mathbf{V} .
\end{aligned}
$$

Therefore

$$
\begin{aligned}
J(\mathbf{Z} \rightarrow \mathbf{V})= & \left|\frac{\partial \operatorname{vec} \mathbf{Z}}{\partial \operatorname{vec}^{\prime} \mathbf{V}}\right| \\
= & \mid\left(\boldsymbol{\Xi}^{-1} \otimes \mathbf{I}_{n}\right)\left\{\boldsymbol{\Delta}^{-1} \otimes \mathbf{I}_{n}+\left(\boldsymbol{\Delta} \otimes \mathbf{I}_{n}\right)\left[\mathbf { K } _ { m n } \left(\mathbf{V}^{\left.+^{\prime} \otimes \mathbf{V}^{+}\right)}\right.\right.\right. \\
& \left.\left.-\left(\mathbf{V}^{\prime} \mathbf{V}\right)^{-1} \otimes\left(\mathbf{I}_{n}-\mathbf{V} \mathbf{V}^{+}\right)\right]\right\} \mid \\
= & |\boldsymbol{\Xi}|^{-n} \mid \boldsymbol{\Delta}^{-1} \otimes \mathbf{I}_{n}+\left(\boldsymbol{\Delta} \otimes \mathbf{I}_{n}\right)\left[\mathbf{K}_{m n}\left(\mathbf{V}^{+^{\prime}} \otimes \mathbf{V}^{+}\right)\right. \\
& \left.-\left(\mathbf{V}^{\prime} \mathbf{V}\right)^{-1} \otimes\left(\mathbf{I}_{n}-\mathbf{V} \mathbf{V}^{+}\right)\right] \mid
\end{aligned}
$$

Alternatively, the Jacobian (11) is expressed in terms of singular values of the matrix $\mathbf{V}$. With this purpose in mind it is used the factorisation of measures.

Lemma 2.1. Let

$$
\mathbf{Y}=\mathbf{U}-\mathbf{U}^{\prime}+
$$

where $\mathbf{Y}$ and $\mathbf{U} \in \Re^{n \times m}$, with element functionally independent, both of rank $m \leq n$. Then

$$
(d \mathbf{Y})=\left\{\begin{array}{l}
\prod_{i=1}^{m}\left(1-l_{i}^{-2}\right)^{n-m}\left(1+l_{i}^{-2}\right) \prod_{i<j}^{m}\left(1-l_{i}^{-2} l_{j}^{-2}\right)(d \mathbf{U}) \\
\prod_{i=1}^{m} l_{i}^{-2 n}\left(l_{i}^{2}-1\right)^{n-m}\left(1+l_{i}^{2}\right) \prod_{i<j}^{m}\left(l_{i}^{2} l_{j}^{2}-1\right)(d \mathbf{U})
\end{array}\right.
$$

where $l_{i}^{2}=\operatorname{ch}_{i}\left(\mathbf{U}^{\prime} \mathbf{U}\right), i=1,2, \ldots, m, l_{1}^{2}>l_{2}^{2}>\cdots>l_{m}^{2}>0$.

Proof. Let $\mathbf{U}=\mathbf{H}_{1} \mathbf{L} \mathbf{Q}^{\prime}$ the singular value factorisation of $\mathbf{U}$, where $\mathbf{H}_{1} \in \mathcal{V}_{m, n}, \mathbf{L}=$ $\operatorname{diag}\left(l_{1}, \ldots, l_{m}\right), l_{1}>\cdots>l_{m}>0$ and $\mathbf{Q} \in \mathcal{O}(m)$, with $l_{i}^{2}=\operatorname{ch}_{i}\left(\mathbf{U}^{\prime} \mathbf{U}\right)$, see Muirhead (2005, Theorem A9.10, p. 593). By Rao (2005, Problem 28e, pp. 76-77) is know that $\mathbf{U}^{+}=\mathbf{Q L}^{-1} \mathbf{H}_{1}^{\prime}$. Then from (13)

$$
\begin{aligned}
\mathbf{Y} & =\mathbf{H}_{1} \mathbf{L} \mathbf{Q}^{\prime}-\left(\mathbf{Q L}^{-1} \mathbf{H}_{1}^{\prime}\right)^{\prime} \\
& =\mathbf{H}_{1}\left(\mathbf{L}-\mathbf{L}^{-1}\right) \mathbf{Q}^{\prime}
\end{aligned}
$$

From Díaz-García and Gutiérrez-Jáimez (2005), taking $g\left(\alpha_{i}\right)=l_{i}-l_{i}^{-1}$ we obtain

$$
(d \mathbf{Y})=\prod_{i=1}^{m}\left(\frac{l_{i}-l_{i}^{-1}}{l_{i}}\right)^{n-m} \prod_{i<j}^{m} \frac{\left(l_{i}-l_{i}^{-1}\right)^{2}-\left(l_{j}-l_{j}^{-1}\right)^{2}}{l_{i}^{2}-l_{j}^{2}} \prod_{i=1}^{m} \frac{d\left(l_{i}-l_{i}^{-1}\right)}{d l_{i}}(d \mathbf{U}) .
$$


Now observe that

$$
\begin{gathered}
\prod_{i=1}^{m} \frac{d\left(l_{i}-l_{i}^{-1}\right)}{d l_{i}}=\left\{\begin{array}{l}
\prod_{i=1}^{m}\left(1+l_{i}^{-2}\right) \\
\prod_{i=1}^{m} l_{i}^{-2} \prod_{i=1}^{m}\left(1+l_{i}^{2}\right),
\end{array}\right. \\
\prod_{i=1}^{m}\left(\frac{l_{i}-l_{i}^{-1}}{l_{i}}\right)^{n-m}=\left\{\begin{array}{l}
\prod_{i=1}^{m}\left(1-l_{i}^{-2}\right)^{n-m} \\
\prod_{i=1}^{m} l_{i}^{-2(n-m)} \prod_{i=1}^{m}\left(l_{i}^{2}-1\right)^{n-m} .
\end{array}\right.
\end{gathered}
$$

Also note that

$$
\begin{aligned}
\left(l_{i}-l_{i}^{-1}\right)^{2}-\left(l_{j}-l_{j}^{-1}\right)^{2} & =\left(\frac{l_{i}^{2}-1}{l_{i}}\right)^{2}-\left(\frac{l_{j}^{2}-1}{l_{j}}\right)^{2} \\
& =\frac{l_{j}^{2}\left(l_{i}^{2}-1\right)^{2}-l_{i}^{2}\left(l_{j}^{2}-1\right)^{2}}{l_{i}^{2} l_{j}^{2}} \\
& =\frac{l_{j}^{2} l_{i}^{4}-2 l_{j}^{2} l_{i}^{2}+l_{j}^{2}-l_{i}^{2} l_{j}^{4}+2 l_{i}^{2} l_{j}^{2}-l_{i}^{2}}{l_{i}^{2} l_{j}^{2}} \\
& =\frac{\left(l_{i}^{2} l_{j}^{2}-1\right)\left(l_{i}^{2}-l_{j}^{2}\right)}{l_{i}^{2} l_{j}^{2}} .
\end{aligned}
$$

From where

$$
\begin{aligned}
\prod_{i<j}^{m} \frac{\left(l_{i}-l_{i}^{-1}\right)^{2}-\left(l_{j}-l_{j}^{-1}\right)^{2}}{l_{i}^{2}-l_{j}^{2}} & =\prod_{i<j}^{m} \frac{\frac{\left(l_{i}^{2} l_{j}^{2}-1\right)\left(l_{i}^{2}-l_{j}^{2}\right)}{l_{i}^{2} l_{j}^{2}}}{l_{i}^{2}-l_{j}^{2}} \\
& =\left\{\begin{array}{l}
\prod_{i=1}^{m} l_{i}^{-2(m-1)} \prod_{i<j}^{m}\left(l_{i}^{2} l_{j}^{2}-1\right) \\
\prod_{i<j}^{m}\left(1-l_{i}^{-2} l_{j}^{-2}\right) .
\end{array}\right.
\end{aligned}
$$

This expression is obtained observing that

$$
\prod_{i<j}^{m} \frac{1}{l_{i}^{2} l_{j}^{2}}=\prod_{i=1}^{m} l_{i}^{-2(m-1)} .
$$

Substituting (16), (17) and (18) into (15) the desired results (14) are obtained.

Theorem 2.2. Under conditions of Theorem 2.1 we have

$$
(d \mathbf{Z})=\frac{1}{|\boldsymbol{\Xi}|^{n}|\boldsymbol{\beta}|^{n / 2}}\left\{\begin{array}{l}
\prod_{i=1}^{m}\left(1-g_{i}^{-2}\right)^{n-m}\left(1+g_{i}^{-2}\right) \prod_{i<j}^{m}\left(1-g_{i}^{-2} g_{j}^{-2}\right)(d \mathbf{V}) \\
\prod_{i=1}^{m} g_{i}^{-2 n}\left(g_{i}^{2}-1\right)^{n-m}\left(1+g_{i}^{2}\right) \prod_{i<j}^{m}\left(g_{i}^{2} g_{j}^{2}-1\right)(d \mathbf{V}),
\end{array}\right.
$$

where $g_{i}^{2}=\operatorname{ch}_{i}\left(\mathbf{V}^{\prime} \mathbf{V} \boldsymbol{\beta}^{-1}\right), i=1, \ldots, m$ and $\boldsymbol{\beta}=\boldsymbol{\Delta}^{2}$. 
Proof. This is immediately by Lemma 2.1 and noting that from (10), $(d \mathbf{Y})=|\boldsymbol{\Xi}|^{n}(d \mathbf{Z})$ and defining $\mathbf{U}=\mathbf{V} \boldsymbol{\Delta}^{-1}$, then $(d \mathbf{U})=|\boldsymbol{\Delta}|^{-n}(d \mathbf{V})=|\boldsymbol{\beta}|^{-n / 2}(d \mathbf{V})$ and $g_{i}^{2}=\operatorname{ch}_{i}\left(\mathbf{U}^{\prime} \mathbf{U}\right)=$ $\operatorname{ch}_{i}\left(\boldsymbol{\Delta}^{-1} \mathbf{V}^{\prime} \mathbf{V} \boldsymbol{\Delta}^{-1}\right)=\operatorname{ch}_{i}\left(\mathbf{V}^{\prime} \mathbf{V} \boldsymbol{\beta}^{-1}\right)$.

\section{Matrix variate generalised Birnbaum-Saunders distri- bution}

This section derives the main result of the paper, the so termed matrix variate generalised Birnbaum-Saunders distribution via a matrix transformation. First we find the distribution of a random matrix $\mathbf{V} \in \Re^{n \times m}$, termed matrix variate square root generalised BirnbaumSaunders distribution, such that $\mathbf{T}=\mathbf{V}^{\prime} \mathbf{V}$ has a matrix variate generalised BirnbaumSaunders distribution; i.e. we shall get the matrix variate version of the density function defined by (7). Then, some special cases are found and, finally basic properties of the matrix variate generalised Birnbaum-Saunders distribution is obtained.

Theorem 3.1. Assume that $\mathbf{Z} \sim \mathcal{E}_{n \times m}\left(\mathbf{0}_{n \times m}, \mathbf{I}_{n m}, h\right)$ and consider the following matrix version of (6)

$$
\mathbf{Z}=\left(\mathbf{V} \boldsymbol{\Delta}^{-1}-\mathbf{V}^{\prime+} \boldsymbol{\Delta}\right) \mathbf{\Xi}^{-1}
$$

where $\boldsymbol{\Xi} \in \Re^{m \times m}, \boldsymbol{\Xi}>\mathbf{0}$ is the shape parameter matrix; $\boldsymbol{\Delta} \in \Re^{m \times m}, \boldsymbol{\Delta}>\mathbf{0}$ is the scale parameter matrix, such that $\boldsymbol{\Delta}$ is the positive definite square root of $\boldsymbol{\beta}\left(\boldsymbol{\Delta}^{2}=\boldsymbol{\beta}\right)$; and $\mathbf{V} \in \Re^{n \times m}$, with $\operatorname{rank}(\mathbf{V})=m \leq n$. Then the density function $d F_{\mathbf{V}}(\mathbf{V})$ of $\mathbf{V}$ is

$$
\begin{aligned}
&=\frac{\mid \boldsymbol{\Delta}^{-1} \otimes \mathbf{I}_{n}}{+}\left(\boldsymbol{\Delta} \otimes \mathbf{I}_{n}\right)\left[\mathbf{K}_{m n}\left(\mathbf{V}^{\prime}+\otimes \mathbf{V}^{+}\right)-\left(\mathbf{V}^{\prime} \mathbf{V}\right)^{-1} \otimes\left(\mathbf{I}_{n}-\mathbf{V} \mathbf{V}^{+}\right)\right] \mid \\
&|\boldsymbol{\Xi}|^{n} \\
& \times h\left[\operatorname{tr} \boldsymbol{\Xi}^{-2}\left(\boldsymbol{\Delta}^{-1} \mathbf{V}^{\prime} \mathbf{V} \boldsymbol{\Delta}^{-1}+\boldsymbol{\Delta}\left(\mathbf{V}^{\prime} \mathbf{V}\right)^{-1} \boldsymbol{\Delta}-2 \mathbf{I}_{m}\right)\right](d \mathbf{V}) .
\end{aligned}
$$

Proof. Define

$$
\mathbf{Z}=\left(\mathbf{V} \boldsymbol{\Delta}^{-1}-\mathbf{V}^{\prime}+\boldsymbol{\Delta}\right) \mathbf{\Xi}^{-1}
$$

then from Theorem 2.1, $d F_{\mathbf{V}}(\mathbf{V})$ is

$$
\begin{array}{r}
=\frac{\left|\boldsymbol{\Delta}^{-1} \otimes \mathbf{I}_{n}+\left(\boldsymbol{\Delta} \otimes \mathbf{I}_{n}\right)\left[\mathbf{K}_{m n}\left(\mathbf{V}^{\prime}+\otimes \mathbf{V}^{+}\right)-\left(\mathbf{V}^{\prime} \mathbf{V}\right)^{-1} \otimes\left(\mathbf{I}_{n}-\mathbf{V} \mathbf{V}^{+}\right)\right]\right|}{|\boldsymbol{\Xi}|^{n}} \\
\times h\left\{\operatorname{tr}\left[\left(\mathbf{V} \boldsymbol{\Delta}^{-1}-\mathbf{V}^{\prime+} \boldsymbol{\Delta}\right) \boldsymbol{\Xi}^{-1}\right]^{\prime}\left[\left(\mathbf{V} \boldsymbol{\Delta}^{-1}-\mathbf{V}^{\prime+} \boldsymbol{\Delta}\right) \boldsymbol{\Xi}^{-1}\right]\right\}(d \mathbf{V}) .
\end{array}
$$

The required result is obtained by noting that $\operatorname{rank}\left(\mathbf{V}^{+} \mathbf{V}\right)=\operatorname{rank}\left(\mathbf{V}^{+}\right)=\operatorname{rank}(\mathbf{V})=m=$ $\operatorname{rank}\left(\mathbf{V}^{\prime} \mathbf{V}\right), \mathbf{V}^{+} \mathbf{V} \in \Re^{m \times m}$ and $\mathbf{V}^{\prime} \mathbf{V} \in \Re^{m \times m}$ then, $\mathbf{V}^{\prime} \mathbf{V}^{\prime}+\left(\mathbf{V}^{+} \mathbf{V}\right)^{\prime}=\mathbf{V}^{+} \mathbf{V}=\mathbf{I}_{m}$ and $\left(\mathbf{V}^{\prime} \mathbf{V}\right)^{+}=\left(\mathbf{V}^{\prime} \mathbf{V}\right)^{-1}$. Then, the desired result is obtained.

In terms of the singular values of $\mathbf{V}$, an alternative expression of (21) is derived in the following result.

Corollary 3.1. Under the hypothesis of Theorem 3.1 the density of matrix variate square root generalised Birnbaum-Saunders distribution $d F_{\mathbf{V}}(\mathbf{V})$ is

$$
=\mathrm{G}\left(g^{2}\right) \quad h\left[\operatorname{tr} \boldsymbol{\Xi}^{-2}\left(\boldsymbol{\Delta}^{-1} \mathbf{V}^{\prime} \mathbf{V} \boldsymbol{\Delta}^{-1}+\boldsymbol{\Delta}\left(\mathbf{V}^{\prime} \mathbf{V}\right)^{-1} \boldsymbol{\Delta}-2 \mathbf{I}_{m}\right)\right](d \mathbf{V}),
$$


where

$$
\mathrm{G}\left(g^{2}\right)=\frac{1}{|\boldsymbol{\Xi}|^{n}|\boldsymbol{\beta}|^{n / 2}}\left\{\begin{array}{l}
\prod_{i=1}^{m}\left(1-g_{i}^{-2}\right)^{n-m}\left(1+g_{i}^{-2}\right) \prod_{i<j}^{m}\left(1-g_{i}^{-2} g_{j}^{-2}\right) \\
\prod_{i=1}^{m} g_{i}^{-2 n}\left(g_{i}^{2}-1\right)^{n-m}\left(1+g_{i}^{2}\right) \prod_{i<j}^{m}\left(g_{i}^{2} g_{j}^{2}-1\right),
\end{array}\right.
$$

with $g_{i}^{2}=\operatorname{ch}_{i}\left(\mathbf{V}^{\prime} \mathbf{V} \boldsymbol{\beta}^{-1}\right), i=1, \ldots, m$.

Proof. This follows straightforwardly from Theorem 2.2 .

The next result define the matrix variate generalised -Saunders distribution via matrix transformation. This fact shall be denoted as

$$
\mathbf{T} \sim \mathcal{G B S}_{m}(n, \boldsymbol{\Xi}, \boldsymbol{\beta}, h),
$$

where $\boldsymbol{\Xi} \in \Re^{m \times m}, \boldsymbol{\Xi}>\mathbf{0}$ is the shape parameter matrix, $\boldsymbol{\Delta} \in \Re^{m \times m}, \boldsymbol{\Delta}>\mathbf{0}$ such that $\boldsymbol{\Delta}$ is the positive definite square root of the scale parameter matrix $\boldsymbol{\beta}$, i.e. $\boldsymbol{\Delta}^{2}=\boldsymbol{\beta}$.

Theorem 3.2. Suppose that $\mathbf{T} \sim \mathcal{G B S}_{m}(n, \boldsymbol{\Xi}, \boldsymbol{\beta}, h), \mathbf{T} \in \Re^{m \times m}, \mathbf{T}>\mathbf{0}, \boldsymbol{\Xi} \in \Re^{m \times m}, \boldsymbol{\Xi}>\mathbf{0}$ and $\boldsymbol{\beta} \in \Re^{m \times m}, \boldsymbol{\beta}>\mathbf{0}$; where $\boldsymbol{\beta}=(\boldsymbol{\Delta})^{2}, \boldsymbol{\Delta}$ is the positive definite square root of $\boldsymbol{\beta}$. Then

$$
\begin{aligned}
d F_{\mathbf{T}}(\mathbf{T})=\frac{\pi^{n m / 2} \mathrm{G}(\delta)}{2^{m} \Gamma_{m}[n / 2]|\boldsymbol{\beta}|^{n / 2}|\boldsymbol{\Xi}|^{n}}|\mathbf{T}|^{(n-m-1) / 2} \\
\quad \times h\left[\operatorname{tr} \boldsymbol{\Xi}^{-2}\left(\boldsymbol{\Delta}^{-1} \mathbf{T} \boldsymbol{\Delta}^{-1}+\boldsymbol{\Delta} \mathbf{T}^{-1} \boldsymbol{\Delta}-2 \mathbf{I}_{m}\right)\right](d \mathbf{T}),
\end{aligned}
$$

where

$$
\mathrm{G}(\delta)=\left\{\begin{array}{l}
\prod_{i=1}^{m}\left(1-\delta_{i}^{-1}\right)^{n-m}\left(1+\delta_{i}^{-1}\right) \prod_{i<j}^{m}\left(1-\delta_{i}^{-1} \delta_{j}^{-1}\right) \\
\prod_{i=1}^{m} \delta_{i}^{-n}\left(\delta_{i}-1\right)^{n-m}\left(1+\delta_{i}\right) \prod_{i<j}^{m}\left(\delta_{i} \delta_{j}-1\right),
\end{array}\right.
$$

where $\delta_{i}=\operatorname{ch}_{i}\left(\boldsymbol{\beta}^{-1} \mathbf{T}\right), i=1, \ldots, m$ and $\Gamma_{m}[\cdot]$ denotes de multivariate gamma function, see Muirhead (2005, Definition 2.1.10, p.61),

$$
\Gamma_{m}[a]=\pi^{m(m-1) / 4} \prod_{i=1}^{m} \Gamma[a-(i-1) / 2],[\operatorname{Re}(a)>(m-1) / 2]
$$

and $\operatorname{Re}(\cdot)$ denotes de real part of the argument.

Proof. By analogy with the univariate case, Equations (5), (6) and (7), starting from (20), we shall say that the positive definite matrix $\mathbf{T}=\mathbf{V}^{\prime} \mathbf{V}$ have a matrix variate generalised Birnbaum-Saunders distribution. In (23), define $\mathbf{T}=\mathbf{V}^{\prime} \mathbf{V}$ with $\mathbf{V}=\mathbf{H}_{1} \mathbf{R}$, where $\mathbf{H}_{1} \in$ $\mathcal{V}_{m, n}$ and $\mathbf{R} \in \Re^{m \times m}$ is a real upper triangular matrix. Then $\mathbf{T}=\mathbf{V}^{\prime} \mathbf{V}=\mathbf{R}^{\prime} \mathbf{R}$. Note that in the considered $\mathrm{QR}$ factorisation $\left(\mathbf{V}=\mathbf{H}_{1} \mathbf{R}\right)$, the matrices $\mathbf{H}_{1}$ and $\mathbf{R}$ are defined in Mathai (1997, p. 100), see Theorem 2.9 and the preceding discussion for the unique choice of $\mathbf{H}_{1}$ and $\mathbf{R}$. Then by Muirhead (2005, Theorem 2.1.14, p. 66)

$$
(d \mathbf{V})=2^{-m}|\mathbf{T}|^{(n-m-1) / 2}(d \mathbf{T})\left(\mathbf{H}_{1}^{\prime} d \mathbf{H}_{1}\right)
$$

Thus, the joint density function of $\mathbf{T}$ and $\mathbf{H}_{1}$ is

$$
d F_{\mathbf{T}, \mathbf{H}_{1}}\left(\mathbf{T}, \mathbf{H}_{1}\right)=\frac{\mathrm{G}(\delta)}{2^{m}|\boldsymbol{\beta}|^{n / 2}|\boldsymbol{\Xi}|^{n}}|\mathbf{T}|^{(n-m-1) / 2}
$$




$$
\times h\left[\operatorname{tr} \boldsymbol{\Xi}^{-2}\left(\boldsymbol{\Delta}^{-1} \mathbf{T} \boldsymbol{\Delta}^{-1}+\boldsymbol{\Delta} \mathbf{T}^{-1} \boldsymbol{\Delta}-2 \mathbf{I}_{m}\right)\right](d \mathbf{T})\left(\mathbf{H}_{1}^{\prime} d \mathbf{H}_{1}\right),
$$

where where

$$
\mathrm{G}(\delta)=\left\{\begin{array}{l}
\prod_{i=1}^{m}\left(1-\delta_{i}^{-1}\right)^{n-m}\left(1+\delta_{i}^{-1}\right) \prod_{i<j}^{m}\left(1-\delta_{i}^{-1} \delta_{j}^{-1}\right) \\
\prod_{i=1}^{m} \delta_{i}^{-n}\left(\delta_{i}-1\right)^{n-m}\left(1+\delta_{i}\right) \prod_{i<j}^{m}\left(\delta_{i} \delta_{j}-1\right)
\end{array}\right.
$$

where $\delta_{i}=\operatorname{ch}_{i}\left(\boldsymbol{\beta}^{-1} \mathbf{T}\right), i=1, \ldots, m$. In this case, see Mathai (1997, p. 117),

$$
\int_{\mathbf{H}_{1}}\left(\mathbf{H}_{1}^{\prime} d \mathbf{H}_{1}\right)=\frac{\pi^{m n / 2}}{\Gamma_{m}[n / 2]}
$$

Where $\Gamma_{m}[\cdot]$ denotes de multivariate gamma function, see Muirhead (2005, Definition 2.1.10, p.61),

$$
\Gamma_{m}[a]=\pi^{m(m-1) / 4} \prod_{i=1}^{m} \Gamma[a-(i-1) / 2],[\operatorname{Re}(a)>(m-1) / 2]
$$

and $\operatorname{Re}(\cdot)$ denotes de real part of the argument. Thus the required result is obtained.

A case of particular interest is when $\boldsymbol{\beta}=\beta \mathbf{I}_{m}, \beta>0$, i.e. when $\mathbf{T} \sim \mathcal{G B S}_{m}\left(n, \boldsymbol{\Xi}, \beta \mathbf{I}_{m}, h\right)$. Note that in this case $\boldsymbol{\Delta}$ such that $\boldsymbol{\beta}=\boldsymbol{\Delta}^{2}$ is $\boldsymbol{\Delta}=\sqrt{\beta} \mathbf{I}_{m}$.

Corollary 3.2. We say that $\mathbf{T} \sim \mathcal{G B S}_{m}\left(n, \boldsymbol{\Xi}, \beta \mathbf{I}_{m}, h\right)$ if its density function is given by

$$
\begin{aligned}
& d F_{\mathbf{T}}(\mathbf{T})=\frac{\pi^{n m / 2} \mathrm{G}(\lambda)}{2^{m} \Gamma_{m}[n / 2] \beta^{n m / 2}|\boldsymbol{\Xi}|^{n}}|\mathbf{T}|^{(n-m-1) / 2} \\
& \times h\left[\operatorname{tr} \boldsymbol{\Xi}^{-2}\left(\frac{1}{\beta} \mathbf{T}+\beta \mathbf{T}^{-1}-2 \mathbf{I}_{m}\right)\right](d \mathbf{T}),
\end{aligned}
$$

where

$$
\mathrm{G}(\lambda)=\left\{\begin{array}{l}
\prod_{i=1}^{m}\left(1-\beta \lambda_{i}^{-1}\right)^{n-m}\left(1+\beta \lambda_{i}^{-1}\right) \prod_{i<j}^{m}\left(1-\beta^{2} \lambda_{i}^{-1} \lambda_{j}^{-1}\right) \\
\beta^{m n} \prod_{i=1}^{m} \lambda_{i}^{-n}\left(\frac{\lambda_{i}}{\beta}-1\right)^{n-m}\left(1+\frac{\lambda_{i}}{\beta}\right) \prod_{i<j}^{m}\left(\frac{\lambda_{i} \lambda_{j}}{\beta^{2}}-1\right),
\end{array}\right.
$$

where $\lambda_{i}=\operatorname{ch}_{i}(\mathbf{T}), i=1, \ldots, m$.

Proof. This follows straightforwardly from Theorem 3.2 .

The Gaussian case is obtained by taking $\mathbf{Z}$ as a matrix variate normal distribution in Theorem 3.1 Hence, from Theorem 2.2 we obtain the matrix variate Birnbaum-Saunders distribution, which shall be denoted as $\mathbf{T} \sim \mathcal{B S}_{m}(n, \boldsymbol{\Xi}, \boldsymbol{\beta})$.

Corollary 3.3. Suppose that $\mathbf{T} \sim \mathcal{B S}_{m}(n, \boldsymbol{\Xi}, \boldsymbol{\beta}), \mathbf{T} \in \Re^{m \times m}, \mathbf{T}>\mathbf{0}, \boldsymbol{\Xi} \in \Re^{m \times m}, \boldsymbol{\Xi}>\mathbf{0}$ and $\boldsymbol{\beta} \in \Re^{m \times m}, \boldsymbol{\beta}>\mathbf{0}$; where $\boldsymbol{\beta}=(\boldsymbol{\Delta})^{2}, \boldsymbol{\Delta}$ is the positive definite square root of $\boldsymbol{\beta}$. Then

$$
\begin{aligned}
d F_{\mathbf{T}}(\mathbf{T})=\frac{\mathrm{G}(\delta)}{2^{m(n+2) / 2} \Gamma_{m}[n / 2]|\boldsymbol{\beta}|^{n / 2}|\mathbf{\Xi}|^{n}}|\mathbf{T}|^{(n-m-1) / 2} \\
\times \operatorname{etr}\left[-\frac{1}{2} \boldsymbol{\Xi}^{-2}\left(\boldsymbol{\Delta}^{-1} \mathbf{T} \boldsymbol{\Delta}^{-1}+\boldsymbol{\Delta} \mathbf{T}^{-1} \boldsymbol{\Delta}-2 \mathbf{I}_{m}\right)\right](d \mathbf{T}),
\end{aligned}
$$


where

$$
\mathrm{G}(\delta)=\left\{\begin{array}{l}
\prod_{i=1}^{m}\left(1-\delta_{i}^{-1}\right)^{n-m}\left(1+\delta_{i}^{-1}\right) \prod_{i<j}^{m}\left(1-\delta_{i}^{-1} \delta_{j}^{-1}\right) \\
\prod_{i=1}^{m} \delta_{i}^{-n}\left(\delta_{i}-1\right)^{n-m}\left(1+\delta_{i}\right) \prod_{i<j}^{m}\left(\delta_{i} \delta_{j}-1\right),
\end{array}\right.
$$

and $\delta_{i}=\operatorname{ch}_{i}\left(\boldsymbol{\beta}^{-1} \mathbf{T}\right)$ and $\operatorname{etr}(\cdot)=\exp (\operatorname{tr}(\cdot))$.

Proof. In the Gaussian case we just take $h(z)=(2 \pi)^{-n m / 2} \operatorname{etr}(-z / 2)$. Then the proof follows straightforwardly from Theorem 2.2

Some basic properties of the matrix variate generalised Birnbaum-Saunders distribution are summarised in the next result.

Theorem 3.3. Assume that $\mathbf{T} \sim \mathcal{G B S}_{m}(n, \boldsymbol{\Xi}, \boldsymbol{\beta}, h)$, then

i) if $\mathbf{S}=\mathbf{T}^{-1}$, its density function is

$$
\begin{aligned}
d F_{\mathbf{S}}(\mathbf{S})=\frac{\pi^{n m / 2} \mathrm{G}(\rho)}{2^{m} \Gamma_{m}[n / 2]|\boldsymbol{\beta}|^{n / 2}|\mathbf{\Xi}|^{n}}|\mathbf{S}|^{-(n+m+1) / 2} & \\
\times & \times h\left[\operatorname{tr} \boldsymbol{\Xi}^{-2}\left(\boldsymbol{\Delta}^{-1} \mathbf{S}^{-1} \boldsymbol{\Delta}^{-1}+\boldsymbol{\Delta} \mathbf{S} \boldsymbol{\Delta}-2 \mathbf{I}_{m}\right)\right](d \mathbf{S}),
\end{aligned}
$$

where

$$
\mathrm{G}(\rho)=\left\{\begin{array}{l}
\prod_{i=1}^{m}\left(1-\rho_{i}^{-1}\right)^{n-m}\left(1+\rho_{i}^{-1}\right) \prod_{i<j}^{m}\left(1-\rho_{i}^{-1} \rho_{j}^{-1}\right) \\
\prod_{i=1}^{m} \rho_{i}^{-n}\left(\rho_{i}-1\right)^{n-m}\left(1+\rho_{i}\right) \prod_{i<j}^{m}\left(\rho_{i} \rho_{j}-1\right),
\end{array}\right.
$$

where $\rho_{i}=\operatorname{ch}_{i}\left(\boldsymbol{\beta}^{-1} \mathbf{S}^{-1}\right), i=1, \ldots, m$.

ii) The density function of $\mathbf{Y}=\mathbf{C}^{\prime} \mathbf{T C}, \mathbf{C} \in \Re^{m \times m}$, non singular, is,

$$
\begin{aligned}
& d F_{\mathbf{Y}}(\mathbf{Y})=\frac{\pi^{n m / 2} \mathrm{G}(\theta)}{2^{m} \Gamma_{m}[n / 2]|\boldsymbol{\beta}|^{n / 2}|\boldsymbol{\Xi}|^{n}|\mathbf{C}|^{n}}|\mathbf{Y}|^{(n-m-1) / 2} \\
& \times h\left[\operatorname{tr} \boldsymbol{\Xi}^{-2}\left((\boldsymbol{\Delta} \mathbf{C})^{\prime-1} \mathbf{Y}(\boldsymbol{\Delta} \mathbf{C})^{-1}+(\boldsymbol{\Delta} \mathbf{C}) \mathbf{Y}^{-1}(\boldsymbol{\Delta} \mathbf{C})^{\prime}-2 \mathbf{I}_{m}\right)\right](d \mathbf{Y}),
\end{aligned}
$$

where

$$
\mathrm{G}(\delta)=\left\{\begin{array}{l}
\prod_{i=1}^{m}\left(1-\theta_{i}^{-1}\right)^{n-m}\left(1+\theta_{i}^{-1}\right) \prod_{i<j}^{m}\left(1-\theta_{i}^{-1} \theta_{j}^{-1}\right) \\
\prod_{i=1}^{m} \theta_{i}^{-n}\left(\theta_{i}-1\right)^{n-m}\left(1+\theta_{i}\right) \prod_{i<j}^{m}\left(\theta_{i} \theta_{j}-1\right),
\end{array}\right.
$$

where $\theta_{i}=\operatorname{ch}_{i}\left(\left(\mathbf{C}^{\prime} \boldsymbol{\beta} \mathbf{C}\right)^{-1} \mathbf{Y}\right), i=1, \ldots, m$.

Proof. The corresponding proofs are obtained by considering the following Jacobians, see Muirhead (2005, Section 2.1.1).

i) Let $\mathbf{S}=\mathbf{T}^{-1}$, then $(d \mathbf{T})=|\mathbf{S}|^{-(m+1)}(d \mathbf{S})$ and

ii) Let $\mathbf{Y}=\mathbf{C}^{\prime} \mathbf{T C}$, then $(d \mathbf{T})=|\mathbf{C}|^{-(m+1)}(d \mathbf{Y})$,

respectively. 


\section{Application}

In this section we study a subfamily of elliptical models usually termed the Kotz type model; given that it includes the Gaussian case, then some interesting comparisons can be made. In our setting, the addressed matrix variate generalised Birnbaum-Saunders distribution based on a Kotz type elliptical model shall be termed matrix variate Kotz-Birnbaum-Saunders distribution and for a Gaussian kernel, the matrix variate Birnbaum-Saunders distribution shall be used.

For parameter estimation and illustration of the distribution here derived, we consider two populations of $K=20$ random symmetric matrices of order 2, measured in certain biology experiment available from the authors. We suppose that the $\mathbf{T}_{k}, k=1, \ldots, K$ matrices are i.i.d matrix variate Kotz-Birnbaum-Saunders. Then, under the Kotz family, the parameters $\beta$ and the elements $\alpha_{11}, \alpha_{12}, \alpha_{22}$ of the matrix $\boldsymbol{\Xi}$ can be estimated via likelihood

First, the density function of the matrix variate Kotz distribution is given by:

$$
d F_{\mathbf{X}}(\mathbf{X})=\frac{s r^{(2 q+n m-2) / 2 s} \Gamma[m n / 2]}{\pi^{m n / 2} \Gamma[(2 q+n m-2) / 2 s]}\left(\operatorname{tr} \mathbf{X}^{\prime} \mathbf{X}\right)^{q-1} \exp \left[-r\left(\operatorname{tr} \mathbf{X}^{\prime} \mathbf{X}\right)^{s}\right](d \mathbf{X})
$$

where $\mathbf{X} \in \Re^{n \times m}, q, r, s \in \Re$, with $r>0, s>0$ and $2 q+m n>2$, see Gupta et al. (2013, p. $54)$.

Now, let $\mathbf{T} \sim \mathcal{G B S}_{m}\left(n, \boldsymbol{\Xi}, \beta \mathbf{I}_{m}, h\right)$, where $h$ is the Kotz kernel, then Corollary 3.2 provides the following density function

$$
\begin{aligned}
d F_{\mathbf{T}}(\mathbf{T})=\frac{s r^{(2 q+n m-2) / 2 s} \Gamma[m n / 2] \mathrm{G}(\lambda)}{2^{m} \Gamma[(2 q+} & n m-2) / 2 s] \Gamma_{m}[n / 2] \beta^{n m / 2}|\mathbf{\Xi}|^{n} \\
\times & |\mathbf{T}|^{(n-m-1) / 2}\left[\operatorname{tr} \boldsymbol{\Xi}^{-2}\left(\frac{1}{\beta} \mathbf{T}+\beta \mathbf{T}^{-1}-2 \mathbf{I}_{m}\right)\right]^{q-1} \\
& \times \exp \left\{-r\left[\operatorname{tr} \boldsymbol{\Xi}^{-2}\left(\frac{1}{\beta} \mathbf{T}+\beta \mathbf{T}^{-1}-2 \mathbf{I}_{m}\right)\right]^{s}\right\}(d \mathbf{T}),
\end{aligned}
$$

where

$$
\mathrm{G}(\lambda)=\left\{\begin{array}{l}
\prod_{i=1}^{m}\left(1-\beta \lambda_{i}^{-1}\right)^{n-m}\left(1+\beta \lambda_{i}^{-1}\right) \prod_{i<j}^{m}\left(1-\beta^{2} \lambda_{i}^{-1} \lambda_{j}^{-1}\right) \\
\beta^{m n} \prod_{i=1}^{m} \lambda_{i}^{-n}\left(\frac{\lambda_{i}}{\beta}-1\right)^{n-m}\left(1+\frac{\lambda_{i}}{\beta}\right) \prod_{i<j}^{m}\left(\frac{\lambda_{i} \lambda_{j}}{\beta^{2}}-1\right),
\end{array}\right.
$$

with $\lambda_{i}=\operatorname{ch}_{i}(\mathbf{T}), i=1, \ldots, m$.

Assuming that $\mathbf{T}_{1}, \ldots \mathbf{T}_{K}$ is a independent random sample, then its likelihood function is given by

$$
\mathrm{L}\left(s, r, q, \beta, \boldsymbol{\Xi} \mid \mathbf{T}_{1}, \ldots \mathbf{T}_{K}\right)=\prod_{k=1}^{K} f_{\mathbf{T}_{1}, \ldots \mathbf{T}_{K}}\left(\mathbf{T}_{1}, \ldots \mathbf{T}_{K} \mid s, r, q, \beta, \boldsymbol{\Xi}\right) .
$$

Explicitly, $\mathrm{L}\left(s, r, q, \beta, \boldsymbol{\Xi} \mid \mathbf{T}_{1}, \ldots \mathbf{T}_{K}\right)$ is

$$
=\frac{s^{K} r^{K(2 q+n m-2) / 2 s}(\Gamma[m n / 2])^{K} \prod_{k=1}^{K} \mathrm{G}\left(\lambda_{k}\right)}{2^{K m}(\Gamma[(2 q+n m-2) / 2 s])^{K}\left(\Gamma_{m}[n / 2]\right)^{K} \beta^{K n m / 2}|\mathbf{\Xi}|^{K n}}
$$




$$
\begin{aligned}
& \times \prod_{k=1}^{K}\left\{\left|\mathbf{T}_{k}\right|^{(n-m-1) / 2}\left[\operatorname{tr} \boldsymbol{\Xi}^{-2}\left(\frac{1}{\beta} \mathbf{T}_{k}+\beta \mathbf{T}_{k}^{-1}-2 \mathbf{I}_{m}\right)\right]^{q-1}\right\} \\
& \times \exp \left\{-r \sum_{k=1}^{K}\left[\operatorname{tr} \boldsymbol{\Xi}^{-2}\left(\frac{1}{\beta} \mathbf{T}_{k}+\beta \mathbf{T}_{k}^{-1}-2 \mathbf{I}_{m}\right)\right]^{s}\right\} .
\end{aligned}
$$

where

$$
\mathrm{G}\left(\lambda_{k}\right)=\left\{\begin{array}{l}
\prod_{i=1}^{m}\left(1-\beta \lambda_{i_{k}}^{-1}\right)^{n-m}\left(1+\beta \lambda_{i_{k}}^{-1}\right) \prod_{i<j}^{m}\left(1-\beta^{2} \lambda_{i_{k}}^{-1} \lambda_{j_{k}}^{-1}\right) \\
\beta^{m n} \prod_{i=1}^{m} \lambda_{i_{k}}^{-n}\left(\frac{\lambda_{i_{k}}}{\beta}-1\right)^{n-m}\left(1+\frac{\lambda_{i_{k}}}{\beta}\right) \prod_{i<j}^{m}\left(\frac{\lambda_{i_{k}} \lambda_{j}}{\beta^{2}}-1\right),
\end{array}\right.
$$

Here $\lambda_{1_{k}}, \ldots, \lambda_{m_{k}}$ are the eigenvalues of $\mathbf{T}_{k}, k=1, \ldots, K$. Then, using logarithms and the first expression for $G\left(\lambda_{k}\right)$, the log-likelihood function,

$$
\mathfrak{L}\left(s, r, q, \beta, \boldsymbol{\Xi} \mid \mathbf{T}_{1}, \ldots \mathbf{T}_{K}\right)=\log \mathrm{L}\left(s, r, q, \beta, \boldsymbol{\Xi} \mid \mathbf{T}_{1}, \ldots \mathbf{T}_{K}\right),
$$

is given as follows

$$
\begin{gathered}
=K \log s+K(2 q+m n-2) / 2 s \log r+K \log \Gamma[m n / 2] \\
+(n-m) \sum_{k=1}^{K} \sum_{i_{k}=1}^{m} \log \left(1-\beta \lambda_{i_{k}}^{-1}\right)+\sum_{k=1}^{K} \sum_{i_{k}=1}^{m} \log \left(1+\beta \lambda_{i_{k}}^{-1}\right) \\
+\sum_{k=1}^{K} \sum_{i_{k}<j_{k}}^{m} \log \left(1-\beta^{2} \lambda_{i_{k}}^{-1} \lambda_{j_{k}}^{-1}\right)-K m \log 2-K \log \Gamma[(2 q+n m-2) / 2 s] \\
-K \log \Gamma_{m}[n / 2]-K n m / 2 \log \beta-K n \log |\boldsymbol{\Xi}|+(n-m-1) / 2 \sum_{k=1}^{K} \log \left|\mathbf{T}_{k}\right| \\
+(q-1) \sum_{k=1}^{K} \log \left[\operatorname{tr} \boldsymbol{\Xi}^{-2}\left(\frac{1}{\beta} \mathbf{T}_{k}+\beta \mathbf{T}_{k}^{-1}-2 \mathbf{I}_{m}\right)\right] \\
-r \sum_{k=1}^{K}\left[\operatorname{tr} \boldsymbol{\Xi}^{-2}\left(\frac{1}{\beta} \mathbf{T}_{k}+\beta \mathbf{T}_{k}^{-1}-2 \mathbf{I}_{m}\right)\right]^{s} .
\end{gathered}
$$

In the application, both populations are based on $n=6$ (BS parameter), $m=2$ (BS dimension) and $K=20$ (sample size).

We require for each population, the MLE of $\beta$ and the three parameters $\alpha_{11}, \alpha_{12}$ and $\alpha_{22}$ in the $2 \times 2$ matrix $\boldsymbol{\Xi}$.

Note that no moment estimators or similar estimates for the parameter matrices in the GBS are available for a plausible starting point of the optimisation algorithm. However, for an initial guess, we can modify in some sense the moment estimation for the two-parameter Birnbaum-Saunders distribution under the univariate Gaussian model given by $\mathrm{Ng}$ et al. (2003). In this case, we use the sample arithmetic and harmonic means for $\alpha_{11}, \alpha_{12}$ and $\alpha_{2}$ of the symmetric matrix $\mathbf{T}_{k}, k=1, \ldots, K$. We also apply the same procedure for the $\beta$ seed.

Computations were based on a number of different methods given in the Optimx package of R. 
In the first population, the following estimates were found for the $2 \times 2$ matrix variate Birnbaum-Saunders distribution under the Gaussian model:

$$
\hat{\beta}=11564.05, \hat{\alpha}_{11}=1.036578, \hat{\alpha}_{12}=0.7515808, \hat{\alpha}_{22}=0.9177609 .
$$

Meanwhile, the corresponding estimations for the second population are given next:

$$
\hat{\beta}=10455.89, \hat{\alpha}_{11}=1.101019, \hat{\alpha}_{12}=0.8329878, \hat{\alpha}_{22}=0.9737600 \text {. }
$$

Recall that the matrix variate Birnbaum-Saunders distribution is a matrix variate KotzBirnbaum-Saunders distribution with parameters $r=1 / 2, q=1$ and $s=1$, then we can compare the results of other matrix variate Kotz-Birnbaum-Saunders distributions. In particular, we fix the parameter $s>0$ in order to follow the performance of the MLE of $r>0$ and $q>(2-m n) / 2$.

Table 1 shows the estimations for the first population.

\begin{tabular}{|c|c|c|c|c|c|c|c|}
\hline$s$ & $\hat{\beta}$ & $\hat{\alpha}_{11}$ & $\hat{\alpha}_{12}$ & $\hat{\alpha}_{22}$ & $\hat{r}$ & $\hat{q}$ & $B I C_{K}^{*}-B I C_{G}^{*}$ \\
\hline 0.5 & 11162.25 & 0.4887122 & 0.3375979 & 0.4352718 & 14.6415 & 47.26912 & 11.31758 \\
\hline 0.75 & 11162.08 & 1.416144 & 0.9781439 & 1.259404 & 12.17327 & 30.5029 & 11.69678 \\
\hline 1 & 11162.10 & 1.845184 & 1.274293 & 1.638595 & 7.487726 & 22.15525 & 12.05738 \\
\hline 1.25 & 11161.98 & 2.926815 & 2.020863 & 2.595519 & 11.14265 & 17.17882 & 12.39958 \\
\hline 1.5 & 11161.96 & 3.380878 & 2.333830 & 2.994185 & 10.99973 & 13.88284 & 12.72398 \\
\hline 1.75 & 11161.95 & 3.697732 & 2.551863 & 3.270593 & 10.93979 & 11.54726 & 13.03058 \\
\hline 2.00 & 11161.94 & 3.917303 & 2.702570 & 3.460524 & 10.89573 & 9.811045 & 13.31978 \\
\hline 3.00 & 11161.93 & 4.311239 & 2.969885 & 3.791473 & 10.81207 & 5.850994 & 14.31638 \\
\hline 4.00 & 11161.92 & 4.407736 & 3.030849 & 3.861463 & 10.80005 & 3.959314 & 15.08558 \\
\hline 5.00 & 11161.91 & 4.418485 & 3.032273 & 3.858097 & 10.79939 & 2.874946 & 15.66898 \\
\hline
\end{tabular}

Table 1: MLE's for some $2 \times 2$ matrix variate Kotz-Birnbaum-Saunders distribution: first population

The second population exhibit notorious different estimations, as it can be checked in Table 2

\begin{tabular}{|c|c|c|c|c|c|c|c|}
\hline$s$ & $\hat{\beta}$ & $\hat{\alpha}_{11}$ & $\hat{\alpha}_{12}$ & $\hat{\alpha}_{22}$ & $\hat{r}$ & $\hat{q}$ & $B I C_{K}^{*}-B I C_{G}^{*}$ \\
\hline 0.5 & 8389.146 & 0.2105478 & 0.1442806 & 0.1851914 & 10.43381 & 99.7684 & 16.81938 \\
\hline 0.75 & 8388.252 & 0.8174268 & 0.5600506 & 0.7190269 & 7.874960 & 64.59744 & 16.68458 \\
\hline 1 & 8387.900 & 1.472571 & 1.0087235 & 1.295358 & 6.285182 & 47.04141 & 16.54578 \\
\hline 1.25 & 8387.725 & 2.074805 & 1.420961 & 1.825156 & 5.560266 & 36.53522 & 16.40258 \\
\hline 1.5 & 8387.625 & 2.581792 & 1.767774 & 2.271147 & 5.178514 & 29.55475 & 16.25518 \\
\hline 1.75 & 8387.563 & 2.989130 & 2.046173 & 2.629435 & 4.931057 & 24.58844 & 16.10378 \\
\hline 2.00 & 8387.521 & 3.30695 & 2.263124 & 2.908916 & 4.721612 & 20.88175 & 15.94838 \\
\hline 3.00 & 8387.468 & 3.981113 & 2.721056 & 3.500900 & 3.782823 & 12.34711 & 15.29098 \\
\hline 4.00 & 8387.454 & 4.253189 & 2.902659 & 3.738150 & 3.076206 & 8.208059 & 14.58658 \\
\hline 5.00 & 8387.444 & 4.459573 & 3.038510 & 3.916646 & 3.046630 & 5.813966 & 13.85258 \\
\hline
\end{tabular}

Table 2: MLE's for some $2 \times 2$ matrix variate Kotz-Birnbaum-Saunders distribution: second population

A number of orders and relations can be inferred from the estimations. However, we focuos on significant differences of the matrix variate Kotz-Birnbaum-Saunders distribution and the matrix variate Birnbaum-Saunders distribution, in both populations. Here we use the well known dimension model theory. In particular we use the modified $B I C^{*}$ criterion of Yang and Yang (2007):

$$
B I C^{*}=-2 \mathfrak{L}(\hat{\Theta}, h)+n_{p}(\log (n+2)-\log 24),
$$

where $\mathfrak{L}(\hat{\Theta}, h)$ is the maximum of the log-likelihood function, $n$ is the sample size and $n_{p}$ is the number of parameters $(\Theta)$ to be estimated for each particular matrix variate KotzBirnbaum-Saunders distribution.

We ask for the best matrix variate generalised Birnbaum-Saunders distribution, referred to the group of the proposed models. The modified $B I C^{*}$ criterion suggests to choose the 
model for which the modified $B I C^{*}$ receives its smallest value. In addition, as proposed by Kass and Raftery (1995) and Raftery (1995), the following selection criteria have been employed in order to compare two contiguous models in terms of its corresponding modified $B I C^{*}$.

Table 3: Grades of evidence corresponding to values of the $B I C^{*}$ difference.

\begin{tabular}{cl}
\hline$B T C^{*}$ difference & Evidence \\
\hline $0-2$ & Weak \\
$2-6$ & Positive \\
$6-10$ & Strong \\
$>10$ & Very strong \\
\hline
\end{tabular}

In these experiments the grades of evidence corresponding to values of the $B I C^{*}$ difference $B I C_{K}^{*}-B I C_{G}^{*}$ are shown in the last column of Tables 1 and 2 Here $\mathrm{K}$ and $\mathrm{G}$ stand for Kotz and Gaussian, respectively.

All the results in both populations attain a very strong difference in favor of the Kotz model. However, population 1 tends to prefer large powers of $s$, instead of population 2, which suggests a small power dominance.

As we usually quote after application of dimension model theory, only the expert in the experiment can provide the underlying model. If the scientist has the knowledge to assume the matrix variate Birnbaum-Saunders distribution, any comparison with a matrix variate Kotz-Birnbaum-Saunders distribution with less $B I C^{*}$ is in fact out of consideration. But, if the matrix variate Birnbaum-Saunders distribution is not accepted for the expert, then strong evidence models are suitable for describing the problem, if the parameters are well interpreted.

Finally, observe that we have assumed an i.i.d sample of Birnbaum-Saunders distributions under an elliptical models, but in general, if the expert expects dependency then the associated likelihood function requires some new insight. This theory is usually elusive in literature, however some translations of a recent work can be explore in future, see Díaz-García et al (2019).

\section{References}

Birnbaum, Z.W., Saunders, S.C., A New Family of Life Distributions, J. Appl. Probab. 6(1969) 637-52.

Balakrishnan, N., Kundu, D., Birnbaum-Saunders distribution: A review of model, analysis and applications (with discussion). Appl. Stoch. Models Bus. Ind. (2018) To appear.

Cadet, A., Polar coordinates in $\mathbf{R}^{n p}$; Application to the computation of the Wishart and beta laws, Sankhyā A 58(1996) 101-113.

Caro-Lopera, F. J., Leiva, V. and Balakrishnan, N., Connection between the Hadamard and matrix products with an application to matrix-variate Birnbaum-Saunders distributions, J. Multivariate Anal. 104 (1)(2012) 126-139.

Caro-Lopera, F. J., Díaz-García, J. A., Diagonalization matrix and its application in distribution theory, Statistics, 50 (4)(2016) 870-880.

Chen, J. J., Novick, M. R., Bayesian analysis for binomial models with generalized beta prior distributions, J. Educational Statist. 9(1984) 163-175. 
Davis, A. W., Invariant polynomials with two matrix arguments, extending the zonal polynomials: Applications to multivariate distribution theory, Ann. Inst. Statist. Math. A31(1979) 465-485.

Desmond, A., Stochastic models of failure in random enviorments, Canad. J. Statist. 13(1985) 171-183.

Díaz-García, J. A., Caro-Lopera, F. J., Pérez Ramírez, F. O., Multivector variate distributions, Sankhyā (2019), Accepted.

Díaz-García, J. A., Gutiérrez-Jáimez, R., Functions of singular random matrices and its applications, TEST 14 (2)(2005) 475-487.

Díaz-García, J. A., Domínguez Molina, J. R., Some generalisations of Birnbaum-Saunders and sinh-normal distributions, Int. Math. Forum. 1 (35)(2006) 1709-1727.

Díaz-García, J. A., Domínguez Molina, J. R., A new family of life distributions for dependent data: Estimation, Comp. Statist. Data Anal. 51(12)(2007) 5927-5939.

Díaz-García, J. A., Leiva-Sánchez, V., A new family of life distributions based on elliptically contoured distributions, J. Statist. Plan. Inf. 128(2)(2005) 445-457.

Díaz-García, J. A., Leiva-Sánchez, V., Erratum to "A new family of life distributions based on the elliptically contoured distributions": [J. Statist. Plann. Inference 128(2) (2005) 445-457], J. Statist. Plan. Inf. 137(4)(2006) 1512-1513.

Fang, K. T., Zhang, Y. T., Generalized Multivariate Analysis, Science Press, SpringerVerlag, Beijing, 1990.

Gupta, A. K., Varga, Y., Bodnar, T., Elliptical Contoured Models in Statistics and Portfolio Theory, Second Edition, Springer, New York, 2013.

Herz, C. S., Bessel Functions of Matrix Argument. Ann. Math. 61(3)(1955) 474-523.

James, A. T., Normal Multivariate Analysis and the Orthogonal Group, Ann Math Statist $25(1)(1954) 40-75$.

Kass R E, Raftery A E (1995) Bayes factor. J Amer Statist Soc 90: 773-795.

Kotz, S., Nadarajah, S., Multivariate $t$ Distributions and Their Applications, Cambridge University Press, United Kingdom, 2004.

Libby, D. L., Novick, M. R., Multivariate generalized beta distributions with applications to utility assessment, J. Educational Statist. 7(1982) 271-294.

Magnus, J. R., Linear Structures, Charles Griffin \& Company Ltd., London, 1988.

Magnus, J. R., Neudecker, H., Matrix differential calculus with application in statistics and econometrics, Third Edition, John Wiley \& Sons, Chichester, 2007.

Mathai, A. M., Jacobian of Matrix Transformations and Functions of Matrix Argument, World Scinentific, Singapore, 1997.

Muirhead, R. J., Aspects of Multivariate Statistical Theory, John Wiley \& Sons, New York, 2005.

Ng, H. K. T., Kundu, D., Balakrishnan, N., 2003. Modified moment estimation for the twoparameter Birnbaum-Saunders distribution, Com. Statist. Data Anal. 43(2003) 283-298. 
Olkin, I., Rubin, H., Multivariate beta distributions and independence properties of Wishart distribution, Ann. Math. Statist. 35(1964) 261-269. Correction 1966, 37(1), 297.

Rao C R (2005) Linear statistical inference and its applications. Second Edition. John Wiley \& Sons, New York

Raftery A E (1995) Bayesian model selection in social research. Sociological Methodology 25: $111-163$.

Roy, S. N. Some aspects of multivariate analysis, John Wiley \& Sons, Inc., New York, 1957.

Sánchez, L., Leiva, V., Caro-Lopera, F., Cysneiros, F.J., On matrix-variate BirnbaumSaunders distributions and their estimation and application. Brazilian J. Probab. Statist. $29(4)(2015) 790-812$

Srivastava, M. S., Khatri, C. G., An Introduction to Multivariate Analysis, North-Holland Publ., Amsterdam, 1979.

Yang Ch Ch, Yang Ch Ch (2007) Separating latent classes by information criteria. J Classification 24: 183-203. 